\title{
CONVEXITY OF PRODUCTION, COMMON POOL AND OLIGOPOLY GAMES: A SURVEY
}

\author{
THEO S. H. DRIESSEN \\ Faculty of Electrical Engineering, Mathematics and Computer Science \\ Department of Applied Mathematics, University of Twente \\ P.O. Box 217, 7500 AE Enschede, The Netherlands \\ E-mail: t.s.h.driessen@math.utwente.nl \\ HOLGER MEINHARDT \\ Institute for Statistics and Economic Theory, University of Karlsruhe \\ Zirkel 2, D-76128 Karlsruhe, Germany \\ E-mail: hme@vwl3.wiwi.uni-karlsruhe.de
}

\begin{abstract}
The paper surveys a uniform proof technique of the convexity property for three different cooperative TU games arising from three different economical settings. The production economy, common pool situation and oligopoly framework involve a cost function, but different production functions. Each of the three corresponding game theoretic models refers to some maximization problem described by optimizing a certain net profit function over all feasible production levels. The current mathematical proof of the convexity of any of three cooperative TU games is strongly based on the interchangeability of maximizers for the underlying maximization problems. This uniform proof technique is inspired by the interchangeability of two players concerning the convexity condition in terms of the marginal contributions of both players in the TU game.
\end{abstract}

1. Introduction. A cooperative game with transferable utility (TU) is a pair $\langle N, v\rangle$, where $N$ is a nonempty, finite set and $v: 2^{N} \rightarrow \mathbb{R}$ is a characteristic function, defined on the power set of $N$, satisfying $v(\emptyset):=0$. An element of $N$ (notation: $i \in N$ ) and a nonempty subset $S$ of $N$ (notation: $S \subseteq N$ or $S \in 2^{N}$ with $S \neq \emptyset$ ) is called a player and coalition respectively, and the associated real number $v(S)$ is called the worth of

2000 Mathematics Subject Classification: Primary 91A12; Secondary 91A40.

JEL Classification: C71.

Key words and phrases: cooperative game, convexity, common pool game, oligopoly game.

The second author gratefully acknowledges financial support by the university of Twente during his research stay at Enschede in November-December 2003.

The paper is in final form and no version of it will be published elsewhere. 
coalition $S$. Concerning the modelling part of game theory, it is customary to investigate whether or not the characteristic function of any appropriate class of cooperative TU games satisfies one or another appealing property. Without going into details, we state that the so-called convexity property of the characteristic function $v$ is a known concept through the game theory literature (cf. [12], [5], [1]). Any cooperative TU game $\langle N, v\rangle$ is said to be a convex game (cf. [12]) if $v(S)+v(T) \leq v(S \cup T)+v(S \cap T)$ for all $S, T \subseteq N$ or equivalently, for all $i \in N, j \in N, i \neq j$, and all $S \subseteq N \backslash\{i, j\}$,

$$
v(S \cup\{i\})+v(S \cup\{j\}) \leq v(S)+v(S \cup\{i, j\}) .
$$

The main purpose of this survey paper is to highlight the uniform approach to establish a new proof of the convexity property for the so-called oligopoly game (to be done in Section 4) similar to the already existing proof of the convexity property for the socalled common pool game (cf. [2], to be recalled in Section 3). The essential part of the forthcoming proof technique is based on the interchangeability of both players $i$ and $j$ concerning the convexity condition (1). That is, this convexity condition (in terms of marginal contributions of two players in the TU game) does not change whenever players $i$ and $j$ are replaced by each other. As an appealing preparation to both forthcoming proofs of higher complexity, we illustrate how some uniform approach works in the less complex framework of a simple production economy (cf. [9]). At the end of Section 3 we outline a uniform approach to prove the convexity property for classes of games arising from maximization problems. The maximizers of any maximization problem are not necessarily unique.

2. The convexity of simple production economy games. Let us start to consider a class of TU games arising from a simple production economy. Any production level is denoted by a non-negative variable $x \in \mathbb{R}_{+}$that is supposed to be not bounded from above. Each participating firm $i \in N$ measures the utility of any production level by its own utility function $u_{i}: \mathbb{R}_{+} \rightarrow \mathbb{R}_{+}$, whereas the costs of production are described by a single cost function $c: \mathbb{R}_{+} \rightarrow \mathbb{R}_{+}$. In the setting of a simple production economy $\left\langle N,\left(u_{i}\right)_{i \in N}, c\right\rangle$, the worth $v(S)$ of any coalition $S$ of firms is determined by some production level that maximizes the sum of the utilities of its members minus its cost of production. Notice that the data of the members of the complementary coalition $N \backslash S$ do not influence the worth of coalition $S$.

Theorem 1 (cf. [9]). Consider a simple production economy $\left\langle N,\left(u_{i}\right)_{i \in N}, c\right\rangle$. The characteristic function $v$ of the associated production economy game $\langle N, v\rangle$ is given by

$$
v(S)=\max _{x \geq 0}\left[\sum_{k \in S} u_{k}(x)-c(x)\right] \quad \text { for all } S \subseteq N, S \neq \emptyset \text {, while } \quad v(\emptyset)=0 .
$$

Without any further assumptions on the cost function and the weakly increasing utility functions, the production economy game $\langle N, v\rangle$ of (2) is a convex game (i.e., (1) holds).

Proof. For all $T \subseteq N, T \neq \emptyset$, let $y_{T} \geq 0$ denote a maximizer for the maximization problem (2) with reference to coalition $T$, that is, $v(T)=\sum_{k \in T} u_{k}\left(y_{T}\right)-c\left(y_{T}\right)$.

Let $i \in N, j \in N, i \neq j$, and $S \subseteq N \backslash\{i, j\}$. Firstly, by the interchangeability of both players $i$ and $j$ concerning the convexity condition (1), we may assume, without loss of 
generality, that the inequality $y_{S \cup\{i\}} \leq y_{S \cup\{j\}}$ holds. That is, in case $y_{S \cup\{i\}} \geq y_{S \cup\{j\}}$, the forthcoming proof applies where the roles of both players $i$ and $j$ are to be interchanged.

Secondly, we exploit that any utility function is supposed to be weakly increasing. Thus, the essential interchangeability inequality $y_{S \cup\{i\}} \leq y_{S \cup\{j\}}$ may be reformulated as another inequality in terms of the utility function $u_{i}$ evaluated at these two maximizers, i.e.,

$$
u_{i}\left(y_{S \cup\{i\}}\right) \leq u_{i}\left(y_{S \cup\{j\}}\right) .
$$

Finally, at the third and final stage, we claim that condition (3) suffices to prove the convexity condition (1) for the production economy game $v$. For that purpose, we consider the two maximizers $y_{S \cup\{i\}}$ and $y_{S \cup\{j\}}$ respectively for the maximization problems (2) with reference to the two augmented coalitions $S \cup\{i\}$ and $S \cup\{j\}$ respectively as feasible production levels for the maximization problems (2) with reference to the two coalitions $S$ and $S \cup\{i, j\}$ respectively in order to bound the worth $v(S)$ and $v(S \cup\{i, j\})$ from below. By these appropriate choices, we arrive at the following (in)equalities:

$$
\begin{aligned}
v(S \cup\{i\}) & =\sum_{k \in S \cup\{i\}} u_{k}\left(y_{S \cup\{i\}}\right)-c\left(y_{S \cup\{i\}}\right), \\
v(S \cup\{j\}) & =\sum_{k \in S \cup\{j\}} u_{k}\left(y_{S \cup\{j\}}\right)-c\left(y_{S \cup\{j\}}\right), \\
v(S) & \geq \sum_{k \in S} u_{k}\left(y_{S \cup\{i\}}\right)-c\left(y_{S \cup\{i\}}\right), \\
v(S \cup\{i, j\}) & \geq \sum_{k \in S \cup\{i, j\}} u_{k}\left(y_{S \cup\{j\}}\right)-c\left(y_{S \cup\{j\}}\right) .
\end{aligned}
$$

In view of (4)-(7), together with (3), it follows immediately that the convexity condition (1) for the production economy game $v$ holds.

Clearly, the above reasoning does not apply if $S=\emptyset$. Fortunately, in case $S=\emptyset$, the corresponding convexity inequality $v(\{i\})+v(\{j\}) \leq v(\{i, j\})$ follows immediately from the maximization problems (2) with reference to the coalitions $\{i\},\{j\}$, and $\{i, j\}$ respectively, by considering the two maximizers $y_{\{i\}}, y_{\{j\}}$ respectively as well as the feasible production level $\max \left[y_{\{i\}}, y_{\{j\}}\right]$ in order to bound the worth $v(\{i, j\})$ from below. This completes the proof of the convexity property (1) for the production economy game of (2). Notice that no property of the cost function is required (except to be non-negative).

\section{The Common Pool Game: the case of a linear cost function}

Definition 1 (cf. $[2,8,4]$ ). Let $N$ be a finite set of individuals (players) and let $w_{i} \geq 0$ denote the endowment of player $i$. For any $T \subseteq N$, denote the total of the endowments by $w_{T}:=\sum_{j \in T} w_{j}$, where $w(\emptyset):=0$. With any joint production function $f: \mathbb{R}_{+} \rightarrow \mathbb{R}_{+}$ satisfying $f(0)=0$ as well as concavity (i.e., $f^{\prime \prime}(x) \leq 0$ for all $x>0$ ), there is associated its weakly decreasing average joint production function $h: \mathbb{R}_{+} \rightarrow \mathbb{R}_{+}$, given by $h(x):=$ $f(x) / x$ for all $x>0$. Further, let $c: \mathbb{R}_{+} \rightarrow \mathbb{R}_{+}$be an arbitrary twice-differentiable cost function satisfying $c(0)=0$.

The corresponding common pool game $\langle N, v\rangle$ is defined such that its player set $N$ consists of the users of the common pool resource and its characteristic function $v$ : 
$2^{N} \rightarrow \mathbb{R}$ is given by $v(\emptyset):=0$ and

$$
v(S):=\max _{0 \leq x \leq w_{S}}\left[x \cdot h\left(x+w_{N \backslash S}\right)-c(x)\right] \quad \text { for all } S \subseteq N, S \neq \emptyset .
$$

THEOREM 2. Let $\langle N, v\rangle$ be the common pool game of (8) supposing the cost function $c$ is linear, i.e., $c(x):=c \cdot x$ for all $x \geq 0$ and a certain constant $c \geq 0$. That is,

$$
v(S):=\max _{0 \leq x \leq w_{S}}\left[x \cdot\left[h\left(x+w_{N \backslash S}\right)-c\right]\right] \quad \text { for all } S \subseteq N, S \neq \emptyset .
$$

Without any further assumptions on the weakly decreasing average joint production function $h$, the common pool game $\langle N, v\rangle$ of (9) is a convex game (i.e., (1) holds).

Proof. For all $T \subseteq N, T \neq \emptyset$, let $y_{T} \in\left[0, w_{T}\right]$ denote a maximizer for the maximization problem (9) with reference to coalition $T$, that is, $v(T)=y_{T} \cdot\left[h\left(y_{T}+w_{N \backslash T}\right)-c\right]$.

Let $i \in N, j \in N, i \neq j$, and $S \subseteq N \backslash\{i, j\}$. Firstly, by the interchangeability of both players $i$ and $j$ concerning the convexity condition (1), we may assume, without loss of generality, that $y_{S \cup\{j\}}-w_{j} \leq y_{S \cup\{i\}}-w_{i}$. That is, in case $y_{S \cup\{j\}}-w_{j} \geq y_{S \cup\{i\}}-w_{i}$, the forthcoming proof applies where the roles of both players $i$ and $j$ are to be interchanged.

Secondly, we exploit that the average joint production function $h$ is supposed to be weakly decreasing. Thus, the essential interchangeability inequality $y_{S \cup\{j\}}-w_{j} \leq$ $y_{S \cup\{i\}}-w_{i}$ may be reformulated as another inequality in terms of the average joint production function $h$ evaluated at certain adapted versions of these two maximizers, i.e.,

$$
h\left(y_{S \cup\{i\}}+w_{N \backslash(S \cup\{i\})}\right) \leq h\left(y_{S \cup\{j\}}+w_{N \backslash(S \cup\{j\})}\right) .
$$

Finally, at the third and final stage, we claim that condition (10) suffices to prove the convexity condition (1) for the common pool game $v$. For that purpose, in addition to the maximizers $y_{S \cup\{i\}}$ and $y_{S \cup\{j\}}$ respectively for the maximization problems (9) with reference to the two augmented coalitions $S \cup\{i\}$ and $S \cup\{j\}$ respectively, we choose the feasible production levels $y_{S \cup\{j\}}+w_{i} \in\left[0, w_{S \cup\{i, j\}}\right]$ as well as $y_{S \cup\{i\}}-w_{i} \in\left[0, w_{S}\right]$ for the maximization problems (9) with reference to the two coalitions $S \cup\{i, j\}$ and $S$ respectively in order to bound the worth $v(S \cup\{i, j\})$ and $v(S)$ from below. By these appropriate choices, we arrive at the following (in)equalities:

$$
\begin{aligned}
v(S \cup\{i\}) & =y_{S \cup\{i\}} \cdot\left[h\left(y_{S \cup\{i\}}+w_{N \backslash(S \cup\{i\})}\right)-c\right], \\
v(S \cup\{j\}) & =y_{S \cup\{j\}} \cdot\left[h\left(y_{S \cup\{j\}}+w_{N \backslash(S \cup\{j\})}\right)-c\right], \\
v(S \cup\{i, j\}) & \geq\left[y_{S \cup\{j\}}+w_{i}\right] \cdot\left[h\left(y_{S \cup\{j\}}+w_{i}+w_{N \backslash(S \cup\{i, j\})}\right)-c\right], \\
v(S) & \geq\left[y_{S \cup\{i\}}-w_{i}\right] \cdot\left[h\left(y_{S \cup\{i\}}-w_{i}+w_{N \backslash S}\right)-c\right] .
\end{aligned}
$$

In view of (11)-(14), together with (10), it follows immediately that the convexity condition (1) for the common pool game $v$ holds.

Clearly, the above reasoning does not apply if $y_{S \cup\{i\}} \leq w_{i}$ (in which case the proposed production level is not feasible). Fortunately, in case $0 \leq y_{S \cup\{i\}} \leq w_{i}$ (which, in turn, covers the subcase $S=\emptyset$ too because of $0 \leq y_{\{i\}} \leq w_{i}$ ), a slightly adapted proof can be presented as follows. Given $0 \leq y_{S \cup\{i\}} \leq w_{i}$ as well as the validity of the assumption (10), it is straightforward to observe that the inequality $v(S \cup\{i\})+v(S \cup\{j\}) \leq v(S \cup\{i, j\})$ already holds since the sum of the right hand sides of (11) and (12) is at most the right 
hand side of (13). This completes the proof of the convexity property (1) for the common pool game assuming a linear cost function.

REMARK 1. Notice that the essential interchangeability assumption $w_{i}-y_{S \cup\{i\}} \leq w_{j}-$ $y_{S \cup\{j\}}$ about the surplus of two maximizers (with respect to the endowments) in the above proof of the convexity for the common pool game can be seen as the counterpart of the essential interchangeability assumption $y_{S \cup\{i\}} \leq y_{S \cup\{j\}}$ about two maximizers in the former proof of the convexity for the production economy game, as dealt within the proof of Theorem 1 in Section 2.

In the context of the study on the convexity property for the common pool game $\langle N, v\rangle$ of (8), it turns out that the general framework needs additional assumptions about the cost function $c$ as well as the average joint production function $h$ (cf. [2]). By Theorem 2, for the special case with a linear cost function, the convexity of the common pool game is valid without any further assumptions on the average joint production function $h$.

REMARK 2. We aim to present a uniform approach to investigate convexity. Consider the underlying game model $\langle N, v\rangle$. Let $i \in N, j \in N, i \neq j$, and $S \subseteq N \backslash\{i, j\}$. Concerning the left hand side of the convexity condition (1), describe the worth of both augmented coalitions $S \cup\{i\}$ and $S \cup\{j\}$ in terms of their corresponding (yet unknown) maximizers (deduced from the objective functions of their corresponding maximization problems). Concerning the right hand side of the convexity condition (1), bound the worth of both coalitions $S$ and $S \cup\{i, j\}$ from below by evaluating the objective function of the two corresponding maximization problems at appropriately chosen adaptations of the former two maximizers. The more complex the game theory model, the more complex to guess such adaptations of the former two maximizers. That is, at the first stage, we replace the convexity condition (1) for the characteristic function $v$ by one sufficient condition with reference to four objective functions, each of one evaluated at (appropriately chosen adaptations of) their maximizers. At the second stage, exploit, as far as possible, the interrelationships among the various objective functions to simplify the former sufficient condition with reference to the objective functions. At the third and final stage, claim that the simplified sufficient condition holds true due to the interchangeability of both players $i$ and $j$ concerning the convexity condition (1). Because of $v(\emptyset)=0$, it is necessary to present a separate proof whenever the coalition $S$ equals the empty set, but fortunately, this separate proof is much less complex.

\section{The Oligopoly Game: the case of linear cost functions}

Definition 2. Let $N$ be a finite set of firms and let $w_{i}>0$ denote the capacity of production by firm $i$. An oligopoly situation $\left\langle N,\left(w_{i}\right)_{i \in N},\left(c_{i}\right)_{i \in N}, a\right\rangle$ with linear cost functions is determined by the capacities $w_{i}, i \in N$, of the firms, their marginal costs $c_{i} \geq 0$, $i \in N$, and the intercept $a \geq 0$ of the inverse demand function $p: \mathbb{R}_{+} \rightarrow \mathbb{R}_{+}$given by $p(z):=\max [0, a-z]$ for all $z \geq 0$. For any $S \subseteq N, S \neq \emptyset$, denote by $\Pi_{S}:=\Pi_{k \in S}\left[0, w_{k}\right]$ the production space of $S$ and write any feasible production schedule $\vec{x}^{S}:=\left(x_{k}\right)_{k \in S} \in \Pi_{S}$ as well as $\vec{x}^{S}(S)=\sum_{k \in S} x_{k}$. 
THEOREM 3. Consider an oligopoly situation $\left\langle N,\left(w_{i}\right)_{i \in N},\left(c_{i}\right)_{i \in N}, a\right\rangle$ with a linear inverse demand function and linear cost functions. The characteristic function $v$ of the corresponding oligopoly game $\langle N, v\rangle$ is defined as follows: $v(\emptyset)=0$ and

$$
v(S)=\max _{\vec{x}^{S} \in \Pi_{S}}\left[\left[a-w(N \backslash S)-\vec{x}^{S}(S)\right] \cdot \vec{x}^{S}(S)-\sum_{k \in S} c_{k} \cdot x_{k}\right] \quad \text { for all } S \subseteq N, S \neq \emptyset .
$$

Without any further assumptions, the oligopoly game $\langle N, v\rangle$ of (15) with a linear inverse demand function and linear cost functions is a convex game (i.e., (1) holds).

Proof. For the sake of notation, for all $T \subseteq N, T \neq \emptyset$, we write $\alpha_{T}:=a-w(N \backslash T)$ as well as the price function $p^{T}\left(\vec{x}^{T}\right):=\alpha_{T}-\vec{x}^{T}(T)$ for all $\vec{x}^{T}=\left(x_{k}\right)_{k \in T} \in \Pi_{T}$. In this setting, the characteristic function $v$, as given by (15), can be reformulated as

$$
v(S)=\max _{\vec{x}^{S} \in \Pi_{S}}\left[p^{S}\left(\vec{x}^{S}\right) \cdot \vec{x}^{S}(S)-\sum_{k \in S} c_{k} \cdot x_{k}\right] \quad \text { for all } S \subseteq N, S \neq \emptyset .
$$

For all $T \subseteq N, T \neq \emptyset$, let $\vec{y}^{T}=\left(y_{k}\right)_{k \in T} \in \Pi_{T}$ denote a maximizer for the maximization problem (16) with reference to coalition $T$, that is $v(T)=p^{T}\left(\vec{y}^{T}\right) \cdot \vec{y}^{T}(T)-\sum_{k \in T} c_{k} \cdot y_{k}$. Let $i \in N, j \in N, i \neq j$, and $S \subseteq N \backslash\{i, j\}$. Concerning the proof of the corresponding convexity property (1), we distinguish two cases: $S=\emptyset$ and $S \neq \emptyset$.

CASE 1. Assume $S=\emptyset$. Instead of $v(\{i\})+v(\{j\}) \leq v(\{i, j\})$, we prove the extended version $v(T)+v(\{i\}) \leq v(T \cup\{i\})$ whenever $T \subseteq N \backslash\{i\}$.

In addition to the maximizers $\vec{y}^{T}$ and $\vec{y}^{\{i\}}$ for the maximization problems (16) with reference to the two coalitions $T$ and $\{i\}$ respectively, we choose the feasible production schedule $\vec{x}^{T \cup\{i\}}=\left(\vec{y}^{T}, \vec{y}^{\{i\}}\right) \in \Pi_{T \cup\{i\}}$ for the maximization problem (16) with reference to coalition $T \cup\{i\}$ in order to bound the worth $v(T \cup\{i\})$ from below. By these appropriate choices, we arrive at the following (in)equalities:

$$
v(\{i\})=p^{\{i\}}\left(\vec{y}^{\{i\}}\right) \cdot \vec{y}^{\{i\}}-c_{i} \cdot \vec{y}^{\{i\}} \quad \text { and } \quad v(T)=p^{T}\left(\vec{y}^{T}\right) \cdot \vec{y}^{T}(T)-\sum_{k \in T} c_{k} \cdot \vec{y}_{k}^{T}
$$

while

$$
v(T \cup\{i\}) \geq p^{T \cup\{i\}}\left(\vec{x}^{T \cup\{i\}}\right) \cdot \vec{x}^{T \cup\{i\}}(T \cup\{i\})-\sum_{k \in T} c_{k} \cdot \vec{y}_{k}^{T}-c_{i} \cdot \vec{y}^{\{i\}} .
$$

In view of the three descriptions, we replace the convexity property $v(T)+v(\{i\}) \leq$ $v(T \cup\{i\})$ for the oligopoly game $v$ by the following weaker, but sufficient condition in which the marginal costs cancel out, while three price functions are left:

$$
p^{T}\left(\vec{y}^{T}\right) \cdot \vec{y}^{T}(T)+p^{\{i\}}\left(\vec{y}^{\{i\}}\right) \cdot \vec{y}^{\{i\}} \leq p^{T \cup\{i\}}\left(\vec{x}^{T \cup\{i\}}\right) \cdot \vec{x}^{T \cup\{i\}}(T \cup\{i\}) .
$$

Clearly, by definition of any price function, the general relationship $\alpha_{T \cup\{i\}}=\alpha_{T}+w_{i}$, and the choice of the schedule $\vec{x}^{T \cup\{i\}}=\left(\vec{y}^{T}, \vec{y}^{\{i\}}\right)$, we get $p^{T \cup\{i\}}\left(\vec{x}^{T \cup\{i\}}\right)=p^{T}\left(\vec{y}^{T}\right)+w_{i}-\vec{y}^{\{i\}}$ and thus, the inequality (17) for price functions reduces to the following inequality:

$$
p^{T}\left(\vec{y}^{T}\right) \cdot \vec{y}^{T}(T)+p^{\{i\}}\left(\vec{y}^{\{i\}}\right) \cdot \vec{y}^{\{i\}} \leq\left[p^{T}\left(\vec{y}^{T}\right)+w_{i}-\vec{y}^{\{i\}}\right] \cdot\left[\vec{y}^{T}(T)+\vec{y}^{\{i\}}\right],
$$

i.e.,

$$
\left[p^{\{i\}}\left(\vec{y}^{\{i\}}\right)-p^{T}\left(\vec{y}^{T}\right)+\vec{y}^{\{i\}}-w_{i}\right] \cdot \vec{y}^{\{i\}} \leq\left[w_{i}-\vec{y}^{\{i\}}\right] \cdot \vec{y}^{T}(T)
$$


Note that $p^{\{i\}}\left(\vec{y}^{\{i\}}\right)-p^{T}\left(\vec{y}^{T}\right)+\vec{y}^{\{i\}}-w_{i}=\vec{y}^{T}(T)-w(T)$ due to $\alpha_{T}-\alpha_{\{i\}}=w(T)-w_{i}$. Hence, the left hand side of the latter inequality (18) is non-positive, whereas its right hand side is non-negative. That is, (18) holds which completes the proof of the convexity property $v(T)+v(\{i\}) \leq v(T \cup\{i\})$ whenever $T \subseteq N \backslash\{i\}$.

CASE 2. Assume $S \neq \emptyset$. In addition to the maximizers $\vec{y}^{S \cup\{i\}}$ and $\vec{y}^{S \cup\{j\}}$ for the maximization problems (16) with reference to the two augmented coalitions $S \cup\{i\}$ and $S \cup\{j\}$ respectively, we choose the feasible production schedules $\vec{x}^{S}:=\left(\vec{y}_{k}^{S \cup\{i\}}\right)_{k \in S} \in \Pi_{S}$ as well as $\vec{x}^{S \cup\{i, j\}}=\left(\left(\vec{y}_{k}^{S \cup\{j\}}\right)_{k \in S \cup\{j\}}, \vec{y}_{i}^{S \cup\{i\}}\right) \in \Pi_{S \cup\{i, j\}}$ for the maximization problems (16) with reference to the two coalitions $S$ and $S \cup\{i, j\}$ respectively in order to bound the worth $v(S)$ and $v(S \cup\{i, j\})$ from below. By these appropriate choices, we arrive at the following (in)equalities:

$$
\begin{gathered}
v(S \cup\{i\})=p^{S \cup\{i\}}\left(\vec{y}^{S \cup\{i\}}\right) \cdot \vec{y}^{S \cup\{i\}}(S \cup\{i\})-\sum_{k \in S \cup\{i\}} c_{k} \cdot \vec{y}_{k}^{S \cup\{i\}}, \\
v(S \cup\{j\})=p^{S \cup\{j\}}\left(\vec{y}^{S \cup\{j\}}\right) \cdot \vec{y}^{S \cup\{j\}}(S \cup\{j\})-\sum_{k \in S \cup\{j\}} c_{k} \cdot \vec{y}_{k}^{S \cup\{j\}}, \\
v(S) \geq p^{S}\left(\vec{x}^{S}\right) \cdot \vec{x}^{S}(S)-\sum_{k \in S} c_{k} \cdot \vec{y}_{k}^{S \cup\{i\}}, \\
v(S \cup\{i, j\}) \geq p^{S \cup\{i, j\}}\left(\vec{x}^{S \cup\{i, j\}}\right) \cdot \vec{x}^{S \cup\{i, j\}}(S \cup\{i, j\}) \\
\quad-\sum_{k \in S \cup\{j\}} c_{k} \cdot \vec{y}_{k}^{S \cup\{j\}}-c_{i} \cdot \vec{y}_{i}^{S \cup\{i\}} .
\end{gathered}
$$

In view of (19)-(22), we replace the convexity condition (1) for the oligopoly game $v$ by the following weaker, but sufficient condition in which the marginal costs cancel out, while four price functions are left:

$$
\begin{aligned}
p^{S \cup\{i\}}\left(\vec{y}^{S \cup\{i\}}\right) \cdot \vec{y}^{S \cup\{i\}}(S \cup\{i\})+p^{S \cup\{j\}}\left(\vec{y}^{S \cup\{j\}}\right) \cdot \vec{y}^{S \cup\{j\}}(S \cup\{j\}) \\
\quad \leq p^{S}\left(\vec{x}^{S}\right) \cdot \vec{x}^{S}(S)+p^{S \cup\{i, j\}}\left(\vec{x}^{S \cup\{i, j\}}\right) \cdot \vec{x}^{S \cup\{i, j\}}(S \cup\{i, j\}) .
\end{aligned}
$$

According to the uniform approach to investigate convexity, at the second stage we are going to simplify the former sufficient condition (23) by exploiting the interrelationships among the four price functions. For the purpose of simplification, we aim to put forward two price functions instead of four. Since $\vec{y}^{S \cup\{i\}}$ is an extension of $\vec{x}^{S}$ as well as $\vec{x}^{S \cup\{i, j\}}$ is an extension of $\vec{y}^{S \cup\{j\}}$, we use the general relationship that, for any augmented production schedule $\vec{z}^{T \cup\{i\}}=\left(\vec{z}^{T}, \vec{z}^{\{i\}}\right) \in \Pi_{T \cup\{i\}}$ it holds:

$$
\begin{aligned}
p^{T \cup\{i\}}\left(\vec{z}^{T \cup\{i\}}\right) \cdot \vec{z}^{T \cup\{i\}}(T \cup\{i\}) \\
\\
=p^{T \cup\{i\}}\left(\vec{z}^{T \cup\{i\}}\right) \cdot \vec{z}^{\{i\}}+p^{T}\left(\vec{z}^{T}\right) \cdot \vec{z}^{T}(T)+\left[w_{i}-\vec{z}^{\{i\}}\right] \cdot \vec{z}^{T}(T)
\end{aligned}
$$

since the definition of the price function applied to $p^{T \cup\{i\}}\left(\vec{z}^{T \cup\{i\}}\right)$ yields $p^{T \cup\{i\}}\left(\vec{z}^{T \cup\{i\}}\right) \cdot\left[\vec{z}^{T}(T)+\vec{z}^{\{i\}}\right]=p^{T \cup\{i\}}\left(\vec{z}^{T \cup\{i\}}\right) \cdot \vec{z}^{\{i\}}+\left[\alpha_{T}+w_{i}-\vec{z}^{T}(T)-\vec{z}^{\{i\}}\right] \cdot \vec{z}^{T}(T)$.

By applying (24) to $T=S$ and $T=S \cup\{j\}$ respectively we derive that the inequality (23) about four price functions reduces to the following inequality about two price functions: 


$$
\begin{aligned}
p^{S \cup\{i\}}\left(\vec{y}^{S \cup\{i\}}\right) \cdot \vec{y}_{i}^{S \cup\{i\}}+\left[w_{i}-\vec{y}_{i}^{S \cup\{i\}}\right] \cdot \vec{y}^{S \cup\{i\}}(S) \\
\leq p^{S \cup\{i, j\}}\left(\vec{x}^{S \cup\{i, j\}}\right) \cdot \vec{y}_{i}^{S \cup\{i\}}+\left[w_{i}-\vec{y}_{i}^{S \cup\{i\}}\right] \cdot \vec{y}^{S \cup\{j\}}(S \cup\{j\})
\end{aligned}
$$

or equivalently,

$$
\begin{aligned}
\vec{y}_{i}^{S \cup\{i\}} \cdot\left[p^{S \cup\{i\}}\left(\vec{y}^{S \cup\{i\}}\right)-p^{S \cup\{i, j\}}\left(\vec{x}^{S \cup\{i, j\}}\right)\right] & \\
& \leq\left[w_{i}-\vec{y}_{i}^{S \cup\{i\}}\right] \cdot\left[\vec{y}^{S \cup\{j\}}(S \cup\{j\})-\vec{y}^{S \cup\{i\}}(S)\right] .
\end{aligned}
$$

Due to the definition of the various price functions, we notice that the following holds:

$$
p^{S \cup\{i\}}\left(\vec{y}^{S \cup\{i\}}\right)-p^{S \cup\{j\}}\left(\vec{y}^{S \cup\{j\}}\right)=w_{i}-w_{j}-\vec{y}^{S \cup\{i\}}(S \cup\{i\})+\vec{y}^{S \cup\{j\}}(S \cup\{j\})
$$

whereas

$$
p^{S \cup\{i\}}\left(\vec{y}^{S \cup\{i\}}\right)-p^{S \cup\{i, j\}}\left(\vec{x}^{S \cup\{i, j\}}\right)=p^{S \cup\{i\}}\left(\vec{y}^{S \cup\{i\}}\right)-p^{S \cup\{j\}}\left(\vec{y}^{S \cup\{j\}}\right)-w_{i}+\vec{y}_{i}^{S \cup\{i\}} .
$$

In view of the latter relationships, the inequality (25) about two non-interchangeable price functions is similar to the following inequality about two interchangeable price functions:

$$
\begin{aligned}
\vec{y}_{i}^{S \cup\{i\}} \cdot\left[p^{S \cup\{i\}}\left(\vec{y}^{S \cup\{i\}}\right)-p^{S \cup\{j\}}\left(\vec{y}^{S \cup\{j\}}\right)-w_{i}+\vec{y}_{i}^{S \cup\{i\}}\right] \\
\leq\left[w_{i}-\vec{y}_{i}^{S \cup\{i\}}\right] \cdot\left[p^{S \cup\{i\}}\left(\vec{y}^{S \cup\{i\}}\right)-p^{S \cup\{j\}}\left(\vec{y}^{S \cup\{j\}}\right)-w_{i}+w_{j}+\vec{y}_{i}^{S \cup\{i\}}\right] .
\end{aligned}
$$

In summary, the convexity condition (1) is replaced by a sufficient condition (23) about four price functions, which in turn is similar to the simplified condition (26) about two interchangeable price functions. At the third and final stage, we claim that the simplified sufficient condition (26) holds true since the left hand side turns out to be nonpositive, whereas the right hand side turns out to be nonnegative. Concerning the right hand side of (26), we claim, without loss of generality, that

$$
p^{S \cup\{i\}}\left(\vec{y}^{S \cup\{i\}}\right)-p^{S \cup\{j\}}\left(\vec{y}^{S \cup\{j\}}\right) \geq w_{i}-w_{j}
$$

due to the interchangeability of both players $i$ and $j$ concerning the convexity condition (1). With respect to the left hand side of (26), we claim that

$$
p^{S \cup\{i\}}\left(\vec{y}^{S \cup\{i\}}\right)-p^{S \cup\{j\}}\left(\vec{y}^{S \cup\{j\}}\right) \leq w_{i}-\vec{y}_{i}^{S \cup\{i\}}
$$

because of the following two monotonicity principles well known in economics:

$$
\begin{array}{cl}
\vec{y}^{S}(S) \leq \vec{y}^{S \cup\{i\}}(S) & \text { (monotonicity of maximizers), } \\
p^{S}\left(\vec{y}^{S}\right) \leq p^{S \cup\{j\}}\left(\vec{y}^{S \cup\{j\}}\right) & \text { (monotonicity of price function at maximizers). }
\end{array}
$$

Given these two types of monotonicity, we derive the inequality (28) as follows:

$$
\begin{aligned}
p^{S \cup\{i\}}\left(\vec{y}^{S \cup\{i\}}\right)-p^{S \cup\{j\}}\left(\vec{y}^{S \cup\{j\}}\right) & =p^{S \cup\{i\}}\left(\vec{y}^{S \cup\{i\}}\right)-p^{S}\left(\vec{y}^{S}\right)+p^{S}\left(\vec{y}^{S}\right)-p^{S \cup\{j\}}\left(\vec{y}^{S \cup\{j\}}\right) \\
& \leq p^{S \cup\{i\}}\left(\vec{y}^{S \cup\{i\}}\right)-p^{S}\left(\vec{y}^{S}\right) \\
& =w_{i}-\vec{y}_{i}^{S \cup\{i\}}+\vec{y}^{S}(S)-\vec{y}^{S \cup\{i\}}(S) \\
& \leq w_{i}-\vec{y}_{i}^{S \cup\{i\}} .
\end{aligned}
$$

This completes the proof of the convexity property (1) of the oligopoly game.

REMARK 3. The interchangeability assumption $p^{S \cup\{i\}}\left(\vec{y}^{S \cup\{i\}}\right)-w_{i} \geq p^{S \cup\{j\}}\left(\vec{y}^{S \cup\{j\}}\right)-w_{j}$ about two maximizers in the above proof of the convexity for the oligopoly game (cf. (27)) can be seen as the counterpart of the essential interchangeability assumption $y_{S \cup\{i\}}-w_{i} \geq$ 
$y_{S \cup\{j\}}-w_{j}$ about two maximizers in the former proof of the convexity for the common pool game (cf. (10) within the proof of Theorem 2 in Section 3). In this context, recall also the essential interchangeability assumption $y_{S \cup\{i\}} \leq y_{S \cup\{j\}}$ about two maximizers in the former proof of the convexity for the production economy game (cf. (3) within the proof of Theorem 1 in Section 2). In summary, the uniform approach to investigate convexity is strongly based on an appropriately chosen interchangeability assumption about two maximizers.

REMARK 4. Concerning the oligopoly games of (15) with a linear inverse demand function and linear cost functions, an alternative proof of its convexity property was established in [10] (as well as in Chapter 3 of the Ph.D. thesis of Kim Hang Pham Do, cf. [11]). In contrast to our uniform approach to investigate convexity, their alternative convexity proof neither refers to maximizers, nor to any essential interchangeability assumption about maximizers. In fact, their alternative convexity proof is extremely tedious, requires a lot of mathematical notation, and its proof technique is based on some complicated induction step to be applied to a slightly adapted oligopoly game. Moreover, the worth of any coalition $S$ in the initial oligopoly game $\langle N, v\rangle$ is treated as the following rather complicated expression:

$$
v(S)=\sum_{k \in S} f_{w_{k}}\left(\max \left[0, a-w(N \backslash S)-c_{k}-2 \cdot \sum_{\ell \in S, \ell<k} w_{\ell}\right]\right) .
$$

Here, for any $x>0$, the associated function $f_{x}: \mathbb{R}_{+} \rightarrow \mathbb{R}$ is given by $f_{x}(y):=\frac{y^{2}}{4}$ for all $0 \leq y \leq 2 \cdot x$, whereas $f_{x}(y):=x \cdot(y-x)$ whenever $y>2 \cdot x$. Altogether, Norde et al.'s proof of convexity for the oligopoly games of (15) is fully based on pure mathematics, whereas our uniform approach to investigate convexity is accessible for a much broader readership, including both economists and mathematicians. In [10], the oligopoly games of (15) are called oligopoly games without transferable technologies. In case it is supposed that every member in a coalition $S$ can produce according to the cheapest technology available in the coalition (that is, the marginal cost $c_{i}$ of any firm $i \in S$ is replaced by $c_{S}:=\min _{k \in S} c_{k}$ ), then so-called oligopoly games with transferable technologies are considered. Necessary and sufficient (but extremely complicated) conditions for the convexity of oligopoly games with transferable technologies are presented in [13].

REMARK 5. In the economic setting of an oligopoly market with $n$ firms producing a homogeneous good, let $w_{i} \geq 0$ represent the production capacity of firm $i$, denote the corresponding cost function by $c: \mathbb{R}_{+} \rightarrow \mathbb{R}_{+}$and let $h: \mathbb{R}_{+} \rightarrow \mathbb{R}_{+}$represent a weakly decreasing inverse demand function. For any $S \subseteq N, S \neq \emptyset$, the profit function $\pi_{S}$ : $\mathbb{R}_{+} \rightarrow \mathbb{R}_{+}$for coalition $S$ is given by $\pi_{S}(x):=x \cdot h\left(x+w_{N \backslash S}\right)-c(x)$ for all $x \geq 0$, where the variable $x$ refers to the production level of coalition $S$ (assuming all the opponents of $S$ produce full capacity). In other words, common pool TU games of (8) reduce to oligopoly games as soon as the average joint production function $h$ can be interpreted as some inverse demand function. Notice that, for the oligopoly games of (8), the production level of coalition $S$ is limited by the total $w_{S}$ of the capacities of its members, whereas for the oligopoly games of (15) the feasible production schedules are limited by the individual capacities $w_{i}, i \in S$, of members of coalition $S$. 
As a particular example of common pool games, let the average joint production function $h$ be an inverse demand function of the form $h(x):=\max [0, a-x]$ for all $x>0$. In other words, the underlying joint production function $f$ is given by a quadratic function, namely $f(x):=x \cdot(a-x)$ for all $0 \leq x \leq a$ and $f(x):=0$ for all $x \geq a$. Most important, $h$ is a weakly decreasing function.

\section{References}

[1] T. S. H. Driessen, Cooperative Games, Solutions, and Applications, Kluwer Academic Publishers, Dordrecht, 1988.

[2] T. S. H. Driessen and H. Meinhardt, (Average) Convexity of common pool and oligopoly TU-games, Int. Game Theory Review 3 (2001), 141-158 (dedicated to contributions to the ICORGT2000 conference held at Chennai, (IIT Madras), January 3-7, 2000).

[3] T. S. H. Driessen and H. Meinhardt, Convexity of oligopoly games without transferable technologies, Math. Social Sci. 50 (2005), 102-126.

[4] Y. Funaki and T. Yamato, The core of an economy with a common pool resource: a partition function form approach, Int. J. Game Theory 28 (1999), 157-171.

[5] E. Iñarra and J. M. Usategui, The Shapley value and average convex games, Int. J. Game Theory 22 (1993), 13-29.

[6] H. Meinhardt, Common pool games are convex games, J. Public Economic Theory 2 (1999), 247-270.

[7] H. Meinhardt, Convexity and k-convexity in cooperative common pool games, Discussion Paper 11, Institute for Statistics and Economic Theory, University of Karlsruhe, Karlsruhe, 1999.

[8] H. Meinhardt, Incentives for cooperative decision making in common pool situations, Ph.D. thesis, Department of Economics, University of Karlsruhe, Karlsruhe, 2000.

[9] H. Moulin, Cores and large cores when population varies, Int. J. Game Theory 19 (1990), 219-232.

[10] H. Norde, K. H. Pham Do and S. H. Tijs, Oligopoly games with and without transferable technologies, Math. Social Sci. 43 (2002), 187-207.

[11] K. H. Pham Do, Essays in game theory and natural resource management, Ph.D. thesis, Dissertation Series nr. 121, CentER for Economic Research, University of Tilburg, Tilburg, 2003.

[12] L. S. Shapley, Cores of convex games, Int. J. Game Theory 1 (1971), 11-26.

[13] J. Zhao, A necessary and sufficient condition for the convexity in oligopoly games, Math. Social Sci. 37 (1999), 189-204. 